\title{
miRNA array analysis of plasma miRNA alterations in rats exposed to a high altitude hypoxic environment
}

\author{
FENG CHEN $^{1,2^{*}}$, REN-JIE WANG ${ }^{3 *}$, GUANG-ZONG LI $^{2 *}$, YI ZHANG ${ }^{2}$, \\ SHUO YU ${ }^{2}$, YING-FU LIU ${ }^{4}$ XIAO-YI CHEN ${ }^{4}$ and SHI-KE HOU ${ }^{1,3}$ \\ ${ }^{1}$ Graduate School, Tianjin Medical University, Tianjin 300070; \\ ${ }^{2}$ Key Laboratory of Disaster and Emergency Rescue Medicine in People's Liberation Army; \\ ${ }^{3}$ Department of Neurosurgery, Logistics University of Chinese People's Armed Police Force, Tianjin 300162; \\ ${ }^{4}$ Department of Cell Biology, Logistics University of Chinese People's Armed Police Forces, Tianjin 300309, P.R. China
}

Received April 12, 2016; Accepted March 20, 2017

DOI: $10.3892 / \mathrm{mmr} .2018 .9570$

\begin{abstract}
In the present study, the microRNA (miRNA) expression profiles of rats exposed to high altitude hypoxia and normal conditions were obtained from miRNA array analysis. Bioinformatics analyses, including the use of the Gene Oncology and Kyoto Encyclopedia of Genes and Genomes databases, were used to identify the genes and pathways, which were specifically associated with high altitude hypoxic environment-associated miRNAs. A total of 26 miRNAs were differentially expressed in the two groups, comprising six upregulated and 20 downregulated miRNAs. In the present study, a novel pattern of upregulated miRNAs and their associated pathways were constructed, including proteoglycans in cancer, spliceosome, gluamatergic synapse, glycolysis/gluconeogenesis, Foxo, cGMP-PKG and p53 signaling pathways, which may provide novel targets for diagnosing and understanding the mechanism of high altitude hypoxia-induced disease.
\end{abstract}

\section{Introduction}

High altitudes create an environment with lower atmospheric pressure, compared with that at sea level. It is reported that there are $\sim 17,000,000$ individuals living 3,500 $\mathrm{m}$ above sea level

Correspondence to: Dr Shi-Ke Hou, Graduate School, Tianjin Medical University, 22 Qixiangtai Road, Heping, Tianjin 300070, P.R. China

E-mail: shikeliu411@163.com

Dr Ying-Fu Liu, Department of Cell Biology, Logistics University of Chinese People's Armed Police Forces, 1 Huizhihuan Road, Dongli, Tianjin 300309, P.R. China

E-mail: liu_yingfu@126.com

*Contributed equally

Key words: microRNA array, rats, high altitude hypoxia, signaling pathways worldwide and encounter hypoxia, which causes deficiency of red blood cells or hemoglobin, and can reduce the ability of the body to transfer oxygen to tissues (1-3). Furthermore, exposure to high altitude can cause digestive system disease, resulting in severe damage to the intestinal tract (4).

MicroRNAs (miRNAs), a set of endogenous small non-coding RNAs with a length of 19-24 nt, exert their functions through translational inhibition or the degradation of target mRNAs (5). It has been demonstrated that miRNAs are key in regulating genes, pathways and various biological networks (5). Circulating miRNAs are being intensively investigated for their involvement in various pathogenic processes and may serve as potential diseases biomarkers $(6,7)$. The concentrations of circulating miRNA can be affected by factors, including age and gender, and environmental factors, including living conditions, residence and altitude (8). However, the expression profile and mechanism of miRNAs under hypoxia at high altitudes remain to be fully elucidated. The advent of genome-wide technologies, including gene expression microarrays, has made it possible to obtain a comprehensive view of miRNA alterations associated with high altitude hypoxia, and the use of bioinformatics enables analysis of the pathways of the altered miRNAs. A previous study (9) found that miRNAs were significantly altered in the Tibetan population, compared with the Nanjing Han population, which suggested that miRNAs may function as important regulators in high altitude hypoxic conditions.

The aim of the present study was to use microarray analysis to examine alterations in the plasma miRNAs of rats exposed to hypobaric hypoxia for different durations. In addition, miRNA functions and pathways were analyzed using Gene Ontology terms and Kyoto Encyclopedia of Genes and Genomes (KEGG) pathway analysis. The results may assist in identifying novel targets to treat high altitude hypoxia-associated disease and provide biomarkers for diagnosis and prognosis.

\section{Materials and methods}

Animals and hypoxic exposure. Adult male Sprague-Dawley rats $(n=24)$ weighing $\sim 180-200 \mathrm{~g}$ were purchased from the Academy of Military Medical Sciences (Beijing, China). The 
animals were maintained in the animal house facility of the Key Laboratory of Disaster and Emergency Rescue Medicine in People's Liberation Army (Tianjin, China) under a standard environment (altitude $0 \mathrm{~m}$, temperature $25 \pm 1^{\circ} \mathrm{C}$, humidity $50 \pm 10 \%$ ) with a $12 \mathrm{~h}$ light/dark cycle, and were provided with food and water for 7 days. To investigate the effects of acute hypobaric hypoxia exposure, the 24 male Sprague-Dawley rats were randomly divided into four groups (I to IV; $n=6$ ). Group I served as the normoxia group maintained in a standard environment. Groups II, III and IV served as hypoxia groups, in which the rats were exposed to simulated hypobaric hypoxia for 24,48 and $72 \mathrm{~h}$, respectively, at 25,000 ft $(8,000 \mathrm{~m} ; 7.5 \mathrm{~mm}$ $\mathrm{Hg} ; 35.4 \mathrm{kPa})$ in a specially designed animal decompression chamber in which altitude was maintained by reducing the ambient barometric pressure, and temperature and humidity were precisely controlled. The airflow in the chamber was $21 / \mathrm{min}$. The temperature and humidity were maintained at $20 \pm 2^{\circ} \mathrm{C}$ and $60 \pm 10 \%$, respectively. The rate of ascent to altitude was maintained at a rate of $300 \mathrm{~m} / \mathrm{min}$ and it took 25-30 min to reach the desired altitude. A slow rate of ascent in altitude with a gradual decrease in ambient pressure was used, as this is less likely to induce decompression-induced gas bubbles during exposure. Experiments and animal care were approved by the ethics committee of the Affiliated Hospital of the Logistics University of Chinese People's Armed Police Force (Tianjin, China).

Blood sample collection. Blood was collected from the inferior cava vein of rats in the normoxia (control) group, and the rats in groups I-IV immediately following 24, 48 and $72 \mathrm{~h}$ exposure to hypoxia. To prepare plasma, anticoagulants (EDTA, heparin or sodium citrate) were added to the blood samples immediately following collection of the blood to prevent clotting. EDTA plasma $(10 \mathrm{ml})$ contained $\sim 1.7 \mathrm{mg}$ potassium EDTA; heparin plasma $(5 \mathrm{ml})$ containing heparin $1 \mathrm{vial}$; sodium citrate plasma, $(10 \mathrm{ml})$ containing $1 \mathrm{ml} 0.118 \mathrm{~mol} / \mathrm{l}$ $(3.2 \%)$ citrate solution. The specimens were then centrifuged at $1,500 \mathrm{~g}$ for $10 \mathrm{~min}$ at $4{ }^{\circ} \mathrm{C}$ to avoid hemolysis, and were then decanted and transferred into RNAase-free Eppendorf tubes as aliquots. The plasma samples were stored at $-80^{\circ} \mathrm{C}$ until further analysis.

Arterial blood measurements. Arterial blood gases were determined using the i-STAT system with the CG8+ cartridge (Abbott Point of Care, Inc., Princeton, NJ, USA). The arterial blood samples $(0.5 \mathrm{ml})$ were collected and the following blood parameters were determined: $\mathrm{pH}$, partial pressure of arterial carbon dioxide $\left(\mathrm{PCO}_{2}\right)$, partial pressure of arterial oxygen $\left(\mathrm{PO}_{2}\right)$, total carbon dioxide $\left(\mathrm{TCO}_{2}\right.$, base excess (BE), saturation of arterial blood oxygen $\left(\mathrm{SaO}_{2}\right)$, bicarbonate concentration $\left(\mathrm{HCO}_{3}^{-}\right)$. The $\mathrm{TCO}_{2}$ was calculated as follows: $\mathrm{TCO}_{2}(\mathrm{mmol} / \mathrm{l})=\mathrm{HCO}_{3}{ }^{-}+0.03 \mathrm{PCO}_{2}$.

Lung wet-to-dry weight (W/D) ratio. Upon termination of each experiment, the animals were sacrificed with a lethal dose of i.v. sodium pentobarbital. The lungs were isolated following chest opening, and the right superior lobe of the hemi-lung was excised and blotted on filter paper to remove adherent blood, with extra pulmonary tissue dissected. The wet weight was determined, followed by drying in an oven at $50^{\circ} \mathrm{C}$ for $72 \mathrm{~h}$ when a constant weight was achieved. The water content of the tissue, which was used as an index of pulmonary edema formation, was calculated as wet weight minus dry weight, expressed as mg water per mg dry tissue.

Lung histology. The remainder of right middle lobe lung was immersed in formalin, embedded in paraffin, cut into $6 \mu \mathrm{m}$ sections and stained with hematoxylin and eosin for histological analysis. The severity of lung injury was inspected using light microscopy (Nikon Eclipse 50i; Nikon Instruments, Inc., Tokyo, Japan) (10).

RNA isolation. TRIzol was used for total RNA extraction according to the manufacturer's protocol (Invitrogen; Thermo Fisher Scientific, Inc., Waltham, MA, USA). Small RNAs ( $<200 \mathrm{nt}$ ) were separated from the total RNA using mirVana miRNA purification columns (Ambion; Thermo Fisher Scientific, Inc.) for microarray analysis and reverse transcription-quantitative polymerase chain reaction (RT-qPCR) analysis according to the manufacturer's protocol. The quality and quantity of each RNA preparation were determined using a Nanodrop ND-1000 spectrophotometer (Agilent Technologies, Inc., Santa Clara, CA, USA).

Expression of miRNAs. Each total RNA sample (700 ng) was labeled and hybridized using a FlashTag ${ }^{\mathrm{TM}}$ Biotin HSR RNA labeling kit (manufactured for Affymetrix, Inc., Santa Clara, CA, USA by Genisphere LLC, Hatfield, PA, USA). Total RNA was labeled using poly A polymerase. Biotin-labeled RNAs were hybridized for $16-18 \mathrm{~h}$ at $45^{\circ} \mathrm{C}$ on an Affymetrix miRNA v2.0 array. GeneChips were washed and stained in the Affymetrix Fluidics Station 450, and were then scanned using the Affymetrix GeneChip Scanner 3000 7G. The data were analyzed using the robust multi-array analysis-detection above background (RMA-DABG) normalization method, implemented in Affymetrix ${ }^{\circledR}$ expression console software (version 1.2.0.20; Affymetrix; Thermo Fisher Scientific, Inc.). The normalized and log-transformed intensity values were analyzed using Expression Console (Affymetrix; Thermo Fisher Scientific, Inc.). Fold change filters were set requiring genes to be present in $\geq 200 \%$ of the controls to be considered an upregulated miRNA and $<50 \%$ of controls to be considered a downregulated miRNA.

$R T$-qPCR analysis. To validate the fold change results of the miRNA arrays, six miRNAs, comprising three upregulated (miR-25-5p, miR-451-5p and miR-466b-5p) and three downregulated (miR-214-3p, miR-140-3p and let-7a-5p) miRNAs, were randomly selected and examined using RT-qPCR analysis. The RT reaction was performed using mature miRNA-specific primer sets (Applied Biosystems; Thermo Fisher Scientific, Inc.) and an microRNA reverse transcription kit (Applied Biosystems; Thermo Fisher Scientific, Inc.) according to the manufacturer's protocol. PCR was performed using the 7500 Fast Real Time PCR system (Applied Biosystems; Thermo Fisher Scientific, Inc.) and the SYBR Premix Ex Taq ${ }^{\mathrm{TM}}$ kit (Takara Biotechnology Co., Ltd., Dalian, China) according to the manufacturer's protocol. The thermocycling conditions were as follows: $95^{\circ} \mathrm{C}$ for $10 \mathrm{~min}$, followed by 35 cycles of $95^{\circ} \mathrm{C}$ for $15 \mathrm{sec}$ and $60^{\circ} \mathrm{C}$ for $1 \mathrm{~min}$. Melting 
Table I. Measurements of arterial blood gas parameters.

High altitude hypoxia (h)

\begin{tabular}{lcccc}
\cline { 3 - 4 } Parameter & Control & 24 & 48 & 72 \\
\hline $\mathrm{pH}$ & $7.396 \pm 0.03$ & $7.369 \pm 0.03$ & $7.291 \pm 0.04$ & $7.263 \pm 0.03$ \\
$\mathrm{PCO}_{2}(\mathrm{mmHg})$ & $34.4 \pm 5.01$ & $37.280 \pm 5.23$ & $39.475 \pm 2.46^{\mathrm{a}}$ & $50.5 \pm 2.89^{\mathrm{a}-\mathrm{c}}$ \\
$\mathrm{PO}_{2}(\mathrm{mmHg})$ & $88.7 \pm 3.98$ & $61.67 \pm 7.12^{\mathrm{a}}$ & $53.33 \pm 2.16^{\mathrm{a}, \mathrm{b}}$ & $41.17 \pm 1.47^{\mathrm{a}-\mathrm{c}}$ \\
$\mathrm{BE}(\mathrm{mmol} / \mathrm{l})$ & $1.67 \pm 0.52$ & $-8 \pm 1.22^{\mathrm{a}}$ & $-8.67 \pm 1.51^{\mathrm{a}}$ & $-9.33 \pm 0.82^{\mathrm{a}}$ \\
$\mathrm{HCO}_{3}(\mathrm{mmol} / \mathrm{l})$ & $30.717 \pm 0.86$ & $19.083 \pm 1.21^{\mathrm{a}}$ & $18.825 \pm 1.33^{\mathrm{a}}$ & $16.25 \pm 0.49^{\mathrm{a}}$ \\
$\mathrm{TCO}_{2}(\mathrm{mmHg})$ & $32.67 \pm 1.51$ & $20 \pm 1.41^{\mathrm{a}}$ & $19.17 \pm 1.94^{\mathrm{a}}$ & $17.5 \pm 1.58^{\mathrm{a}}$ \\
$\mathrm{SaO}_{2}(\%)$ & $91.67 \pm 1.86$ & $67.5 \pm 1.8^{\mathrm{a}}$ & $57 \pm 2.37^{\mathrm{a}, \mathrm{b}}$ & $47.8 \pm 2.48^{\mathrm{a}-\mathrm{c}}$ \\
$\mathrm{Lac}(\mathrm{mmol} / \mathrm{l})$ & $0.982 \pm 0.36$ & $1.488 \pm 0.72$ & $1.537 \pm 0.74$ & $1.635 \pm 0.81$ \\
\hline
\end{tabular}

${ }^{\mathrm{a}} \mathrm{P}<0.01$, compared with the control group; ${ }^{\mathrm{b}} \mathrm{P}<0.01$, compared with the $24 \mathrm{~h}$ group; ${ }^{\mathrm{c}} \mathrm{P}<0.01$, compared with the $48 \mathrm{~h}$ group $(\mathrm{n}=6)$. $\mathrm{PCO}$, partial pressure of arterial carbon dioxide; $\mathrm{PO}_{2}$, partial pressure of arterial oxygen; $\mathrm{BE}$, base excess; $\mathrm{HCO}_{3}^{-}$, bicarbonate; $\mathrm{TCO}_{2}$, total carbon dioxide; $\mathrm{SaO}_{2}$, saturation of arterial blood oxygen; Lac, lactate.

curve analyses were subsequently performed. The fold change for each miRNA was calculated using the $2^{-\Delta \Delta \mathrm{Cq}}$ method (11) with U6 small nuclear RNA as the endogenous control. All reactions were performed in triplicate for each sample. The PCR primer sequences were as follows: The universal reverse primer was: CCAGTGCAGGGTCCGAGGT; and the forward primer sequences were as follows: miR-25-5p, TGCGGAGGC GGAGACUUGGG; miR-451-5p, TGCGGAAACCGUUAC CAUUA; miR-466b-5p, TGCGGUGAUGUGUGUGUACA; miR-214-3p, TGCGGACAGCAGGCACAGAC; miR-140-3p, TGCGGUACCACAGGGUAGAA; let-7a-5p, TGCGGUGAG GUAGUAGGUUG; and U6, TGCGGGTGCTCGCTTCGG CAGC.

MicroRNA target prediction. The miRNA target sites were predicted by computer-aided algorithms obtained from TargetScan (version 7.1; www.targetscan.org/). The miRNA sequences were downloaded from the miRBase website (http:// www.mirbase.org).

Gene ontology (GO) analysis. Based on the GO database (http://www.geneontology.org), the significant GO terms of the upregulated miRNA targeted genes were analyzed using the Database for Annotation, Visualization and Integrated Discovery (http://david.abcc.ncifcrf.gov/home.jsp) (12). Fisher's exact test and the Chi-square test were used to classify the GO categories, and the false discovery rate (FDR) was calculated to correct the P-values using the Benjamini and Hochberg procedure (13). The P-values of each differentially expressed gene in all the GO terms were calculated. $\mathrm{P}<0.05$ was considered to indicate a statistically significant difference.

Pathway analysis. Pathway analysis was performed to determine the significant pathways of the differentially expressed genes, according to the KEGG Orthology Based Annotation System (http://kobas.cbi.pku.edu.cn/), in which pathway enrichment can be analyzed by the KEGG pathway, pathway interaction database, BioCyc, Reactome and Protein ANalysis
THrough Evolutionary Relationships. Fisher's exact test and a $\chi^{2}$ test were used to select the significant pathway, and the threshold of significance was defined by the P-value and false discovery rate (13).

Statistical analysis. Numerical data are presented as the mean \pm standard deviation. Differences between means were analyzed using Student's t-test. For comparison of multiple groups, one-way analysis of variance followed by Tukey's post-test was performed. All statistical analyses were performed using SPSS 13.0 software (SPSS, Inc., Chicago, IL, USA).

\section{Results}

Blood gas analysis. The results of the blood gases analysis confirmed that, compared with control group, $\mathrm{PCO}_{2}$ and lactate were markedly increased in the high altitude hypoxia group, whereas $\mathrm{PO}_{2}, \mathrm{BE}, \mathrm{HCO}_{3}^{-}, \mathrm{TCO}_{2}$ and $\mathrm{SaO}_{2}$ were significantly decreased at 24, 48 and $72 \mathrm{~h}$ in a time-dependent manner $(\mathrm{P}<0.01)$. The $\mathrm{pH}$ level showed marginal decrease with no statistically significant difference (Table I).

Lung W/D ratio and histology. The W/D lung weight ratio was measured to evaluate fluid accumulation in the lung specimens (14). Compared with the normal control groups, the W/D of lung tissues in the hypoxia groups were significantly upregulated at 24,48 and $72 \mathrm{~h}(\mathrm{P}<0.01)$. The W/D of the lung tissues at 48 and $72 \mathrm{~h}$ were higher, compared with that at $24 \mathrm{~h}$. However, no significant difference in the lung W/D ratio was observed between the 48 and $72 \mathrm{~h}$ time points (Fig. 1A). Using light microscopy, a detailed examination of lung pathology was performed. Compared with the control groups, the pulmonary interstitium showed marginal dilations or hyperemia in the $24 \mathrm{~h}$ group. In the $48 \mathrm{~h}$ group, the pulmonary interstitium exhibited increased dilations and thickening. Blood stasis was also identified in the alveolar space. The tissues in the $72 \mathrm{~h}$ group exhibited marked thickening of the pulmonary interstitium and visible pink exudant in the alveolar space. 
Table II. List of miRNAs with altered expression in high altitude hypoxia.

\begin{tabular}{ll}
\hline miRNA & Fold change \\
\hline Upregulated & \\
rno-miR-25-3p & 6.796382 \\
rno-miR-451-5p & 6.811991 \\
rno-miR-466b-5p & 2.243331 \\
rno-miR-486 & 3.692567 \\
rno-miR-879-3p & 3.723761 \\
rno-miR-92a-3p & 2.373798 \\
Downregulated & \\
rno-let-7a-5p & -7.25955 \\
rno-let-7c-5p & -3.42339 \\
rno-miR-125a-3p & -2.86652 \\
rno-miR-140-3p & -5.06208 \\
rno-miR-1896 & -3.84228 \\
rno-miR-208a-5p & -2.92098 \\
rno-miR-214-3p & -4.88104 \\
rno-miR-22-3p & -7.2768 \\
rno-miR-223-3p & -6.82643 \\
rno-miR-23a-3p & -7.45505 \\
rno-miR-24-3p & -6.97121 \\
rno-miR-27a-3p & -2.41551 \\
rno-miR-2985 & -8.11875 \\
rno-miR-3573-3p & -5.68544 \\
rno-miR-483-3p & -4.86634 \\
rno-miR-494-3p & -2.27509 \\
rno-miR-6216 & -8.4358 \\
rno-miR-6328 & -7.31127 \\
rno-miR-672-5p & -5.84322 \\
rno-miR-762 & -3.6636 \\
\hline & \\
\hline & \\
\hline
\end{tabular}

miRNA/miR, microRNA.

Hyperemia and expansion of the alveolar capillary were also observed (Fig. 1B).

Overview of the miRNAs profiles. From the miRNAs expression profiles, differentially expressed miRNAs were identified between the high-altitude hypoxia environment and normal control samples. The miRNA expression profiles were determined by calculating the log FC in the high-altitude hypoxia group/normal group. Among a set of differentially expressed miRNAs, six miRNAs were significantly upregulated and 20 miRNAs were significantly downregulated in the high-altitude hypoxia group, compared with the control group. miR-451-5p, miR-25-3p, miR-879-3p, miR-486, miR-92a-3p and miR-466b-5p were the identified upregulated miRNAs, whereas miR-6216, miR-2985, miR-23a-3p, miR-6328, miR-22-3p and let-7a-5p were the top six significantly downregulated miRNAs (Fig. 2 and Table II).

Validation of the microarray analysis data using $R T-q P C R$ analysis. The relative expression changes of the randomly selected miRNAs were analyzed using RT-qPCR analysis. The results of the miR-25-5p, miR-451-5p and miR-466b-5p upregulated miRNAs, and the miR-214-3p, miR-140-3p and let-7a-5p the downregulated miRNAs were generally consistent with the microarray analysis results, as shown in the histograms in Fig. $3(\mathrm{P}<0.05)$.

Microarray-based GO analysis. For GO analysis, the target mRNAs for the six upregulated miRNAs were predicted using TargetScan (data not shown). As shown in Fig. 4A-D, $\mathrm{GO}$ analysis showed that the targets were involved in several biological processes, and the top five gene-associated processes were cellular process, single-organism process, metabolic process, biological process and regulation of biological process (Fig. 4A). Several genes were involved in the cellular component, the top five of which were cell, cell part, organelle, membrane and organelle part (Fig. 4B). Several genes were also involved in molecular function, the top five of which were binding, catalytic activity, molecular transducer activity, transporter activity and molecular function regulator (Fig. 4C and D). These results supported the hypothesis that these biological processes, cellular components and molecular functions are important in rats exposed to high altitude hypoxia.

Microarray-based pathway analysis. Pathway-based analysis assists in further understanding the biological functions of genes. In the present study, KEGG pathway enrichment analysis was performed to identify significantly enriched metabolic pathways or signal transduction pathways in the differentially expressed genes. Using pathway analysis, which considered the relative change direction and fold change, and had a the threshold of significance of $\mathrm{P}<0.05$, the top 30 significant pathways were identified (Fig. 5). The pathways targeted by upregulated miRNAs with the highest enrichment included the proteoglycans in cancer, spliceosome, gluamatergic synapse, glycolysis/gluconeogenesis, Foxo, cGMP-PKG and p53 signaling pathways, which suggested that these pathways may be involved in the response to a high altitude hypoxic environment.

\section{Discussion}

Understanding the clinical relevance of miRNA expression patterns in the environment during exposure to high altitude is necessary to circumvent the therapeutic challenges faced in clinical management. The present study used bioinformatics methods to screen the differently expressed miRNAs, and then analyzed the functions and pathways of the upregulated miRNAs. In the present study, a total of 26 aberrantly expressed miRNAs were identified in the blood of rats exposed to high altitude, compared to the samples from rats in normal conditions. Subsequently, RT-qPCR analysis was used to confirm the miRNA array results. To measure the concentrations of miRNAs, three upregulated and three downregulated miRNAs were selected for RT-qPCR analysis, the results of which were in accordance with the miRNA array assays. Among the differently expressed miRNAs, a subset of the altered miRNAs (miR-24, miR-25, miR-486, miR-451 and miR-92a) has also been associated with the hypoxic response 

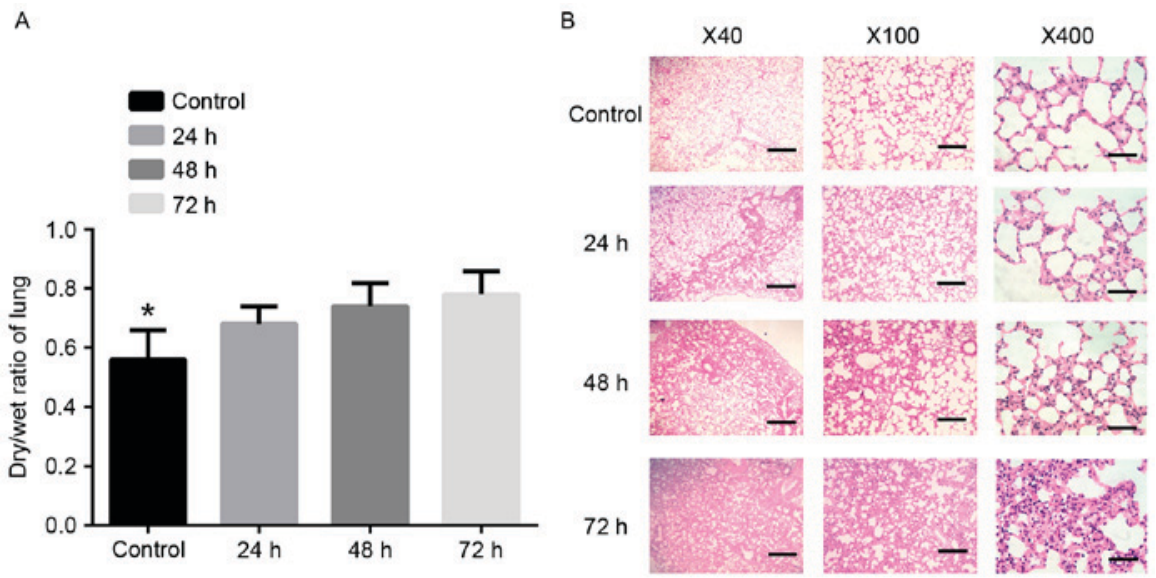

Figure 1. Lung pathology. (A) Wet-to-dry lung weight ratios were measured in the normal control group and high-altitude hypoxia groups at 24,48 and $72 \mathrm{~h}$. ${ }^{*} \mathrm{P}<0.01$, vs. 24, 48 and $72 \mathrm{~h}$ groups. (B) Light micrograph of hematoxylin and eosin-stained paraffin-embedded sections.
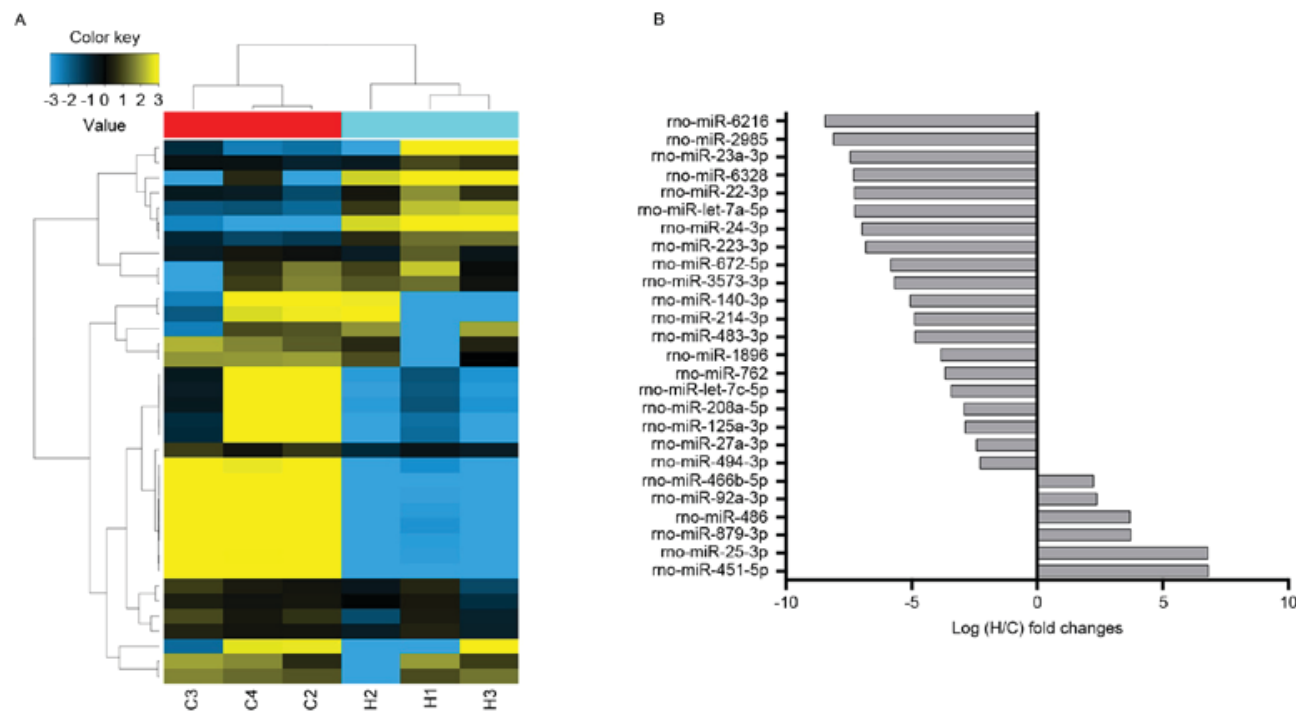

Figure 2. (A) Hierarchical clustering analysis of miRNA expression in rats exposed to high altitude hypoxic and normal conditions. miRNAs are presented in rows and samples are presented in columns. Colors indicate relative signal intensities, the color key value indicates the fold-change. (B) Column graph shows the fold changes of the differently expressed miRNAs. miRNA, microRNA.
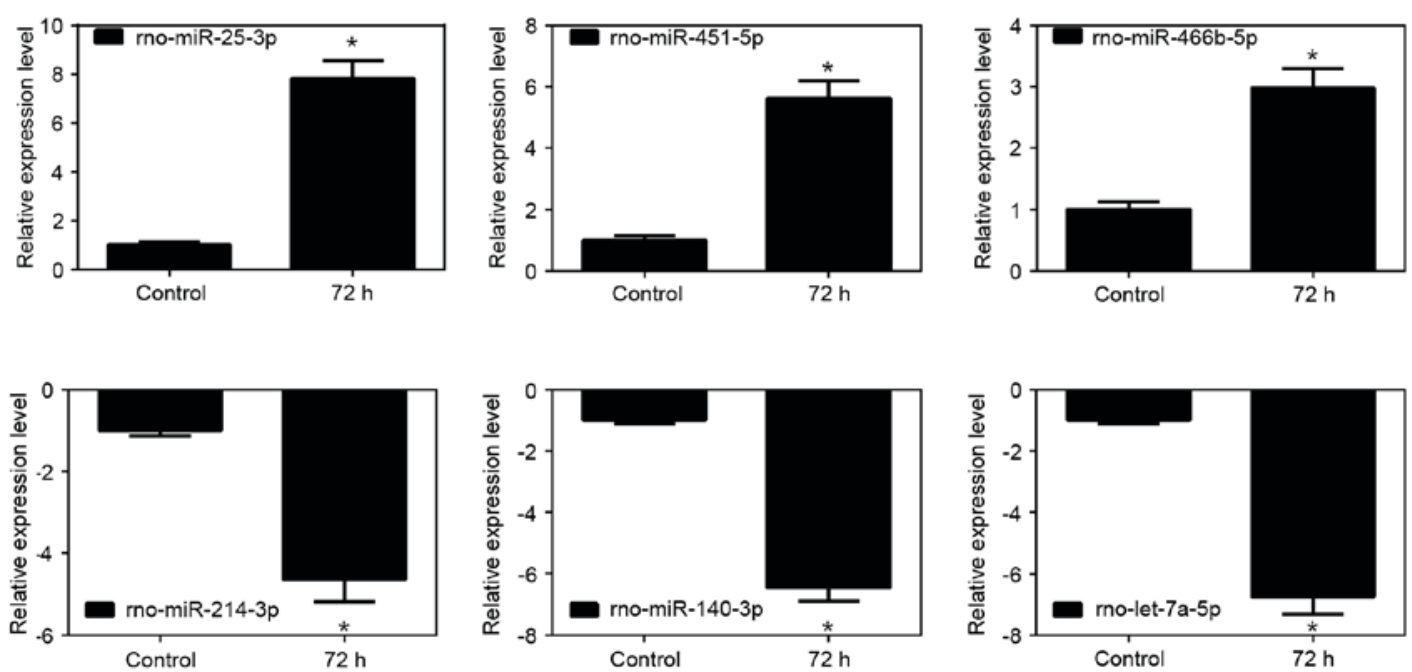

Figure 3. Summary of the results of the reverse transcription-quantitative polymerase chain reaction analyses of upregulated miRNAs (miR-25-3p, miR-451-5p and miR-466b-5p) and downregulated miRNAs (miR-214-3p, miR-140-3p and let-7a-5p). The six randomly selected miRNAs in the control and hypoxia groups are shown with relative expression levels shown on the $y$-axis as upregulated and downregualated. ${ }^{~} \mathrm{P}<0.05$, vs. control. miRNA/miR, microRNA. 

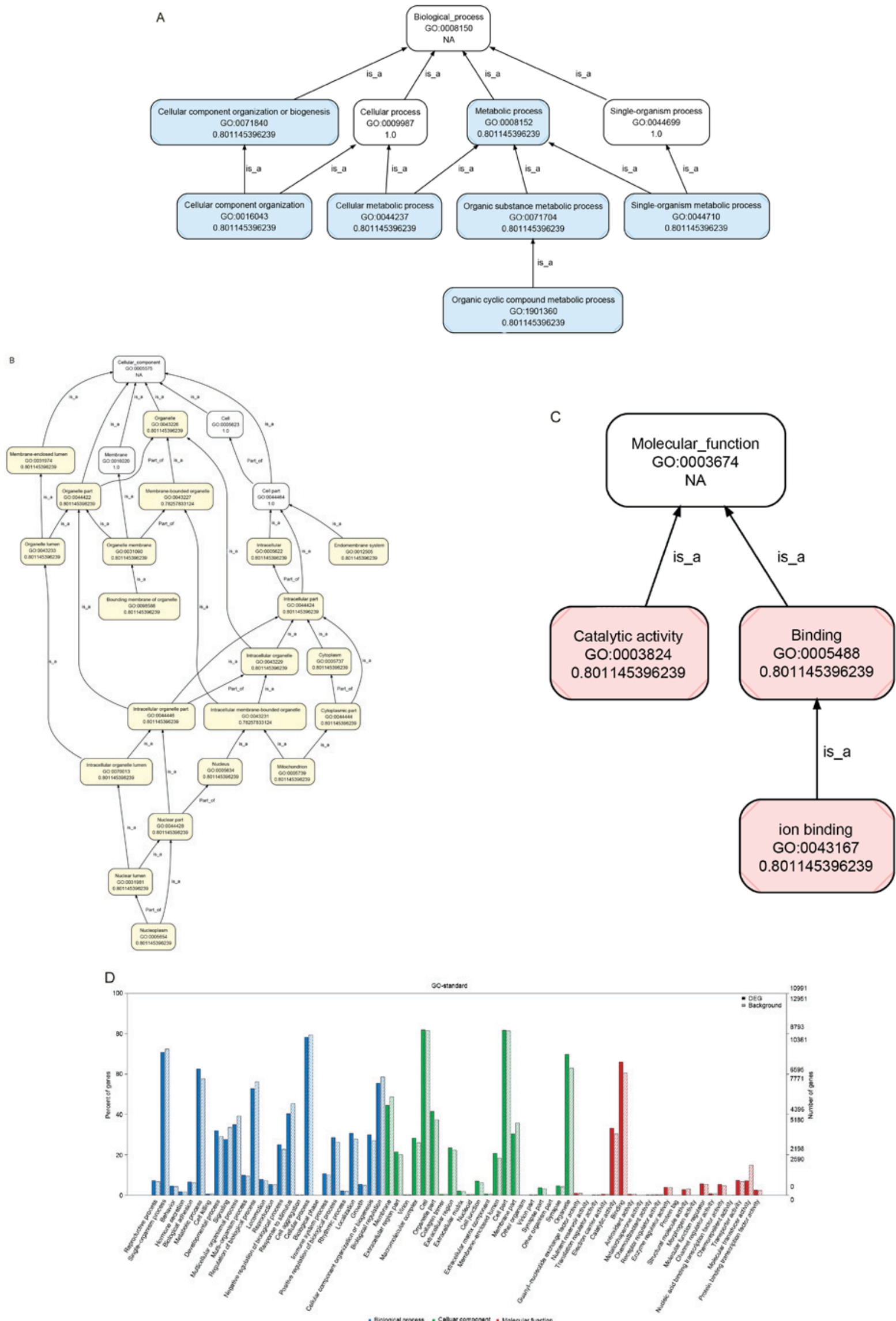

Figure 4. GO analysis of differentially expressed mRNAs in response to the high altitude hypoxic environment. The most related parts are shown for (A) biological process, (B) cellular component, (C) molecular function and (D) percentage of genes associated with the GO terms. GO, Gene Ontology. The node number indicates the corrected $\mathrm{P}$-value $(\mathrm{P}<0.001)$. The node color indicates the Benjamini and Hochberg method corrected $\mathrm{P}$-value for the enrichment of the term; the blue indicates biological process, yellow indicates cellular component, and red indicates molecular function, while the white color indicates little statistical enrichment. 


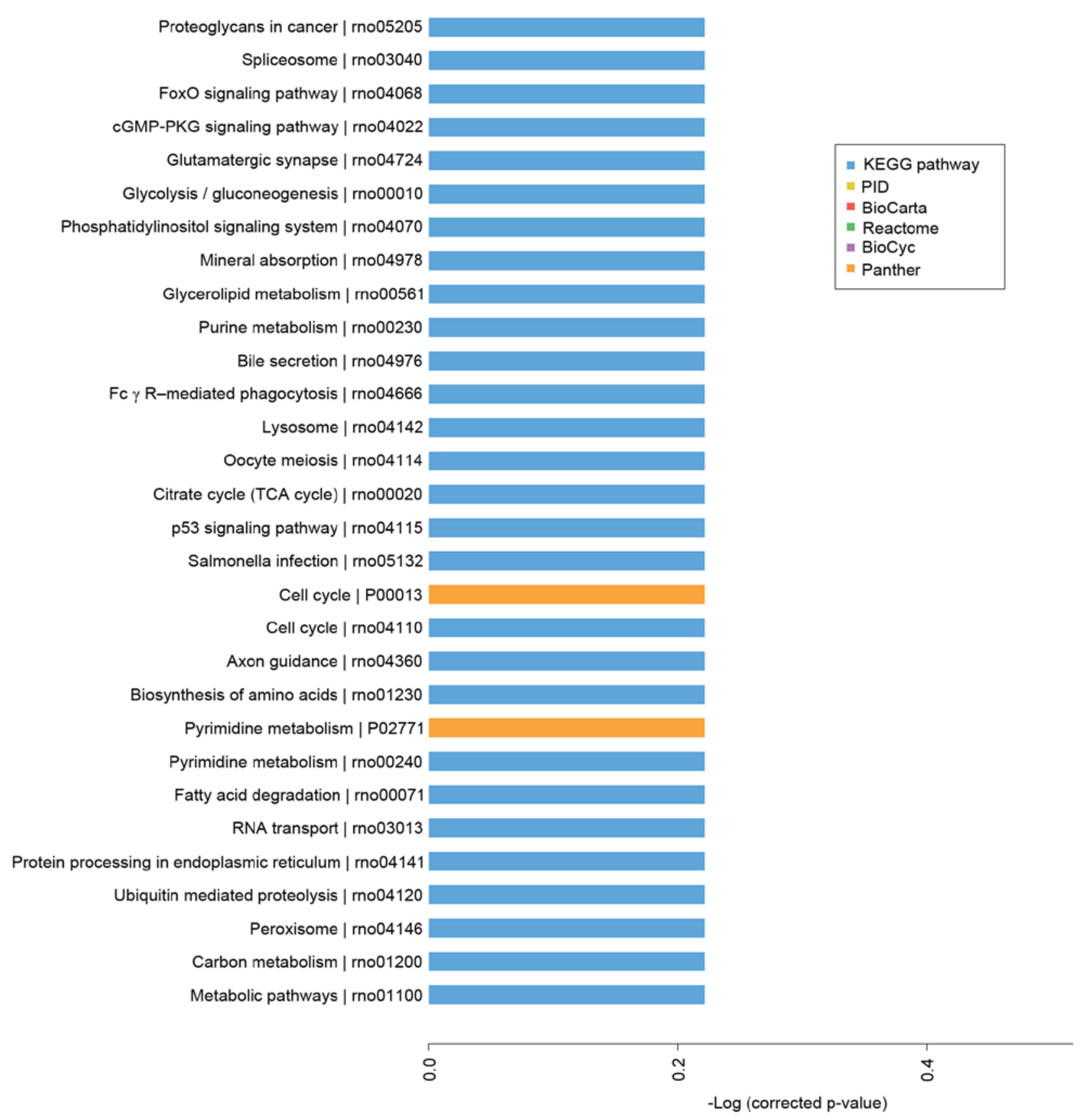

Figure 5. Histogram of signaling pathways found to differ significantly between the high altitude hypoxia and normal environments. The x-axis shows the negative logarithm of the P-value $(\log P)$, the y-axis shows the pathway. The higher the Log P, the lower the P-value.

in mammalian cells in other studies (15-19), which suggested that a high altitude hypoxic environment has a marked effect on human plasma miRNA patterns. The functional investigation of miRNAs, which respond during hypoxia, may assist in uncovering the molecular basis of hypoxic acclimatization and elucidate the complexity of hypoxic response pathways in humans. The present study also found that the expression of rno-miR-23a-3p was similar with the that reported in a study by Yan et al (9), which showed that human miR-23a-3p was downregulated in the Tibetan population, compared with the Nanjing Han population. This suggested the species conservation of this miRNA and indicated its importance for further investigation.
GO is widely recognized as a premier tool for molecular organization and functional annotation (20). Using the criteria of $\mathrm{P}<0.05$ to identify significant GO terms, the present study revealed that the predicted targets of the six upregulated miRNAs were associated with biological processes, including cellular process, single-organism process, metabolic process, biological process and regulation of biological process; cellular components, including cell, cell part, organelle, membrane and organelle part; and molecular functions, including binding, catalytic activity, molecular transducer activity, transporter activity and molecular function regulator. The above GO terms have also been well represented in Triplophysa dalaica (21). 
Pathway analysis can reveal distinct biological processes and identify the significant pathways, which dysregulated mRNAs are involved in. This enables a comprehensive understanding of the interactions of genes, their functions and the association between upstream and downstream genes, and can identify genes, which may be regulated by miRNAs. The appearance of the pathways in proteoglycans in cancer, spliceosome, gluamatergic synapse, glycolysis/gluconeogenesis, Foxo, cGMP-PKG and p53 signaling pathways confirmed their concordance with GO terms and their critical role in high altitude hypoxia. A significant adaptation was identified as an increased evolutionary rate and positive selection of genes involved in the hypoxic response and energy metabolism, analogous to those observed in other organisms in Tibet (22). It was previously reported that a conversion from oxidative glucose metabolism to glycolysis compensated for insufficient levels of oxygen in hypoxic conditions (23-25). The present study also showed the enhanced role of glycolysis. The FOXO subfamily of Forkhead transcription factors has a role in evolutionary conservation in cellular adaptation to stress stimuli, including hypoxic conditions (26). A previous study by Wang et al (27) showed that FOXO1 may be essential in adaptation to high altitudes. p53, as an upstream mediator of p21, may suppress hypoxic human lung fibroblast proliferation and pulmonary arterial remodeling by interacting with hypoxia-inducible factor-1 under hypoxia $(28,29)$. A previous study showed that p53 gene deficiency with a decreased expression of $\mathrm{p} 21$ promoted hypoxia-induced pulmonary hypertension in mice (30). cGMP-dependent protein kinase (PKG) is a critical enzyme involved in the regulation of vascular contractility. Impaired PKG-mediated signaling has been found to be responsible for reduced cGMP-mediated pulmonary hypertension and vasodilatation following acute and chronic hypoxia (31-33). As these pathways have been identified to be involved in various hypoxic environments, together with the results of the present study, these pathways may be critical in high altitude hypoxic conditions.

In conclusion, the results of the present study identified six upregulated miRNAs and 20 downregulated miRNAs from two platforms. As upregulated miRNAs may better serve as biomarkers, the six upregulated miRNAs were used to perform GO and pathway analysis, which identified that Foxo, cGMP-PKG and p53 may be critical in the study model of hypoxia. Based on the integrated analysis of transcriptome features, these results may provide an important contribution to future investigations aimed at characterizing the role of specific miRNAs in the pathogenesis of high altitude hypoxia-induced diseases, and contribute to improving diagnosis and treatment.

\section{Acknowledgements}

Not applicable.

\section{Funding}

This study was funded by the Science and technology planning project of Tianjin City (grant no. 14ZCDZSY00033), the Opening fund Key laboratory of Disaster \& Emergency Rescue Medicine in People's Liberation Army (grant no.
JY1402), the Central laboratory opening fund of Logistics University of Chinese People's Armed Police Forces (grant no. 2015ZXKF01), the Technical project of logistics equipment of Logistics University of Chinese People's Armed Police Forces (grant no. WHZ201507), the National Natural Science Foundation of China (grant no. 81772018), the Natural Science Foundation of Tianjin City (grant no. 17JCZDJC35400) and the Opening fund of Affiliated Hospital of Logistics University of Chinese People's Armed Police Force (grant no. WYKFZ201603).

\section{Availability of data and materials}

The analyzed datasets generated during the study are available from the corresponding author on reasonable request.

\section{Authors' contributions}

FC, RJW and GZL performed the experiments. YZ, SY and XYC analyzed the data. YFL and SKH designed the experiment. SKH drafted the manuscript and revised it critically to produce the final approval of the version to be published.

\section{Ethics approval and consent to participate}

Experiments and animal care were approved by the ethics committee of the Affiliated Hospital of the Logistics University of Chinese People's Armed Police Force (Tianjin, China).

\section{Patient consent for publication}

Not applicable.

\section{Competing interests}

The authors declare that they have no competing interests.

\section{References}

1. Sherpa LY, Deji, Stigum H, Chongsuvivatwong V, Thelle DS and Bjertness E: Obesity in Tibetans aged 30-70 living at different altitudes under the north and south faces of Mt. Everest. Int J Environ Res Public Health 7: 1670-80, 2010.

2. Windsor JS and Rodway GW: Rodway, Heights and haematology: The story of haemoglobin at altitude. Postgrad Med J 83: 148-151, 2007.

3. Peyssonnaux C, Nizet V and Johnson RS: Role of the hypoxia inducible factors HIF in iron metabolism. Cell Cycle 7: 28-32, 2008.

4. Recavarren-Arce S, Ramirez-Ramos A, Gilman RH, Chinga-Alayo E, Watanabe-Yamamoto J, Rodriguez-Ulloa C, Miyagui J, Passaro DJ and Eza D: Severe gastritis in the Peruvian Andes. Histopathology 46: 374-379, 2005.

5. Bartel DP: MicroRNAs: Genomics, biogenesis, mechanism, and function. Cell 116: 281-297, 2004.

6. Mitchell PS, Parkin RK, Kroh EM, Fritz BR, Wyman SK, Pogosova-Agadjanyan EL, Peterson A, Noteboom J, O'Briant KC, Allen A, et al: Circulating microRNAs as stable blood-based markers for cancer detection. Proc Natl Acad Sci USA 105: 10513-10518, 2008.

7. Chen X, Ba Y, Ma L, Cai X, Yin Y, Wang K, Guo J, Zhang Y, Chen J, Guo X, et al: Characterization of microRNAs in serum: A novel class of biomarkers for diagnosis of cancer and other diseases. Cell Res 18: 997-1006, 2008.

8. Meder B, Backes C, Haas J, Leidinger P, Stähler C, Großmann T, Vogel B, Frese K, Giannitsis E, Katus HA, et al: Influence of the confounding factors age and sex on microRNA profiles from peripheral blood. Clin Chem 60: 1200-1208, 2014. 
9. Yan Y, Shi Y, Wang C, Guo P, Wang J, Zhang CY and Zhang C: Influence of a high-altitude hypoxic environment on human plasma microRNA profiles. Sci Rep 5: 15156, 2015.

10. Su X, Song Y, Jiang J and Bai C: The role of aquaporin-1 (AQP1) expression in a murine model of lipopolysaccharide-induced acute lung injury. Respir Physiol Neurobiol 142: 1-11, 2004.

11. Livak KJ and Schmittgen TD: Analysis of relative gene expression data using real-time quantitative PCR and the 2(-Delta Delta C(T)) method. Methods 25: 402-408, 2001.

12. Dennis G Jr, Sherman BT, Hosack DA, Yang J, Gao W, Lane HC and Lempicki RA: DAVID: Database for annotation, visualization, and integrated discovery. Genome Biol 4: P3, 2003.

13. Dupuy D, Bertin N, Hidalgo CA, Venkatesan K, Tu D Lee D, Rosenberg J, Svrzikapa N, Blanc A, Carnec A, et al: Genome-scale analysis of in vivo spatiotemporal promoter activity in Caenorhabditis elegans. Nat Biotechnol 25: 663-668, 2007.

14. Michel RP, Hakim TS, Smith TT and Poulsen RS: Quantitative morphology of permeability lung edema in dogs induced by alpha-naphthylthiourea. Lab Invest 49: 412-419, 1983.

15. Kulshreshtha R, Ferracin M, Wojcik SE, Garzon R, Alder H, Agosto-Perez FJ, Davuluri R, Liu CG, Croce CM Negrini M, et al: A microRNA signature of hypoxia. Mol Cell Biol 27: 1859-1867, 2007.

16. Liang H, Studach L, Hullinger RL, Xie J and Andrisani OM Down-regulation of RE-1 silencing transcription factor (REST) in advanced prostate cancer by hypoxia-induced miR-106b 25. Exp Cell Res 320: 188-199, 2014.

17. Caruso P, MacLean MR, Khanin R, McClure J, Soon E, Southgate M, MacDonald RA, Greig JA, Robertson KE, Masson R, et al: Dynamic changes in lung microRNA profiles during the development of pulmonary hypertension due to chronic hypoxia and monocrotaline. Arterioscler Thromb Vasc Biol 30: 716-723, 2010.

18. Shi XF, Wang H, Xiao FJ, Yin Y, Xu QQ, Ge RL and Wang LS: MiRNA-486 regulates angiogenic activity and survival of mesenchymal stem cells under hypoxia through modulating Akt signal. Biochem Biophys Res Commun 470: 670-677, 2016.

19. Zhang B, Zhou M, Li C, Zhou J, Li H, Zhu D, Wang Z, Chen A and Zhao Q: MicroRNA-92a inhibition attenuates hypoxia/reoxygenation-induced myocardiocyte apoptosis by targeting Smad7. PLoS One 9: e100298, 2014.

20. Lovering RC, Camon EB, Blake JA and Diehl AD: Access to immunology through the Gene Ontology. Immunology 125 154-160, 2008.

21. Wang Y, Yang L, Wu B, Song Z and He S: Transcriptome analysis of the plateau fish (Triplophysa dalaica): Implications for adaptation to hypoxia in fishes. Gene 565: 211-220, 2015.
22. Yang Y, Wang L, Han J, Tang X, Ma M, Wang K, Zhang X, Ren Q, Chen Q and Qiu Q: Comparative transcriptomic analysis revealed adaptation mechanism of Phrynocephalus erythrurus, the highest altitude Lizard living in the Qinghai-Tibet Plateau. BMC Evol Bio 15: 101, 2015

23. Butler PJ and Jones DR: Physiology of diving of birds and mammals. Physiol Rev 77: 837-899, 1997.

24. Denko NC: Hypoxia, HIF1 and glucose metabolism in the solid tumour. Nat Rev Cancer 8: 705-713, 2008

25. Semenza GL: Hypoxia-inducible factors in physiology and medicine. Cell 148: 399-408, 2012.

26. Bakker WJ, Harris IS and Mak TW: FOXO3a is activated in response to hypoxic stress and inhibits HIF1-induced apoptosis via regulation of CITED2. Mol Cell 28: 941-953, 2007.

27. Wang B, Zhang YB, Zhang F, Lin H, Wang X, Wan N, Ye Z, Weng $\mathrm{H}$, Zhang $\mathrm{L}$, Li X, et al: On the origin of Tibetans and their genetic basis in adapting high-altitude environments. PLoS One 6: e17002, 2011

28. Yu J, Liu XW and Kim HR: Platelet-derived growth factor (PDGF) receptor-alpha-activated c-Jun NH2-terminal kinase-1 is critical for PDGF-induced p21WAF1/CIP1 promoter activity independent of p53. J Biol Chem 278: 49582-49588, 2003.

29. Mizuno S, Bogaard HJ, Voelkel NF, Umeda Y, Kadowaki M, Ameshima S, Miyamori I and Ishizaki T: Hypoxia regulates human lung fibroblast proliferation via p53-dependent and -independent pathways. Respir Res 10: 17, 2009.

30. Mizuno S, Bogaard HJ, Kraskauskas D, Alhussaini A, Gomez-Arroyo J, Voelkel NF and Ishizaki T: p53 Gene deficiency promotes hypoxia-induced pulmonary hypertension and vascular remodeling in mice. Am J Physiol Lung Cell Mol Physiol 300: L753-L761, 2011.

31. Gao Y, Dhanakoti S, Trevino EM, Sander FC, Portugal AM and Raj JU: Effect of oxygen on cyclic GMP-dependent protein kinase-mediated relaxation in ovine fetal pulmonary arteries and veins. Am J Physiol Lung Cell Mol Physiol 285: L611-L618, 2003.

32. Gao Y, Portugal AD, Negash S, Zhou W, Longo LD and Usha Raj J: Role of Rho kinases in PKG-mediated relaxation of pulmonary arteries of fetal lambs exposed to chronic high altitude hypoxia. Am J Physiol Lung Cell Mol Physiol 292: L678-L684, 2007.

33. Jernigan NL and Resta TC: Chronic hypoxia attenuates cGMP-dependent pulmonary vasodilation. Am J Physiol Lung Cell Mol Physiol 282: L1366-L1375, 2002.

This work is licensed under a Creative Commons Attribution-NonCommercial-NoDerivatives 4.0 International (CC BY-NC-ND 4.0) License. 